\title{
A Formal Concept Analysis of Harmonic Forms and Interval Structures
}

\author{
Tobias Schlemmer and Stefan E. Schmidt \\ Technische Universität Dresden, \\ Fachrichtung Mathematik, 01062 Dresden, Germany \\ Tobias.Schlemmer@mailbox.tu-dresden.de, midt1@msn.com \\ http://www.math.tu-dresden.de/ schlemme/
}

\begin{abstract}
While small concept lattices are often represented by line diagrams to better understand their full structure, large diagrams may be too complex to do this. However, such a diagram may still be used to receive new ideas about the inherent structure of a concept lattice. This will be demonstrated for a certain family of formal contexts arising from mathematical musicology. In particular, we investigate how chord patterns can be characterised by their interval structure. For such contexts of pattern structures, it turns out that each corresponding concept lattice incorporates two competing building principles, one emanating from the top the other from the bottom of the lattice.
\end{abstract}

Key words: formal concept lattice, harmonic form, musicology, interval

\section{Introduction}

Harmonic forms provide basic notions for the descriptions of chords. Well-known examples are the harmonic form of a major triad which stands for all major chords and, similarly, the minor triad which is the harmonic form of minor chords.

Besides harmonies and chords, harmonic forms play an important role for tuning software like Mutabor [1]. In general, applications of mathematical musicology to music software unfold different questions about the mathematical structure of harmonic forms in tone systems.

In the past, formal concept analysis has been applied to various fields of music already (e.g. [3], [4], [5], and [6]). Here as well as in other fields of mathematical musicology (cf. Mazzola et al. [7]), harmonic forms have been analysed to a certain extent, however, there are still plenty of open problems to address.

The description of the structure of harmonic forms leads to concept lattices that are often considered as too large to be drawn meaningfully. However, the diagrams will serve us as a source of information useful for finding more adequate mathematical models.

(C) Radim Belohlavek, Sergei O. Kuznetsov (Eds.): CLA 2008, pp. 9-22, ISBN 978-80-244-2111-7, Palacký University, Olomouc, 2008. 


\section{Qualitative analysis of harmonic forms}

To describe musical objects, we need mathematical notions of them. A fundamental one is that of a tone system, which can be modelled as a collection of tones and their interval structure (see also [9], [10]):

A triple $\mathrm{T}=(T, \delta, \mathrm{I})$ is called an (algebraic) tone system, if $T$ is a set, $\mathrm{I}=(I,+, 0)$ is an Abelian group and $\delta: T \times T \rightarrow I$ is a map such that for all $t_{1}, t_{2}, t_{3} \in T$ the following hold:

$$
\begin{gathered}
\delta\left(t_{1}, t_{2}\right)+\delta\left(t_{2}, t_{3}\right)=\delta\left(t_{1}, t_{3}\right) \text { and } \\
\delta\left(t_{1}, t_{2}\right)=0 \text { iff } t_{1}=t_{2} .
\end{gathered}
$$

The elements of the set $T$ are called tones and each subset of $T$ is called a chord. The elements of $I$ are considered as intervals. For $s, t \in T$, the interval from $s$ to $t$ is given by $i \in I$ if $i=\delta(s, t)$ holds; in this case we can agree upon $s+i:=t$. For a tone $t$, the set of all intervals from $t$ is given by $I(t):=\delta[\{t\} \times T]$; it follows $t+I(t)=T$ and $I(s)=\delta(s, t)+I(t)$ for all $s, t \in T$.

We call $\mathrm{T}$ homogeneous if $I(t)$ is a subgroup of I for some tone $t$; in this case, we observe that $I(s)=I(t)=\delta[T \times T]$ holds for all tones $s$ and $t$ in T. We refer to $\mathrm{T}$ as a (freely) $n$-generated tone system if $\mathrm{T}$ is homogeneous, $\delta[T \times T]=I$, and $I$ is a (freely) $n$-generated group.

A transposition by the interval $i \in I$ is defined as the map $\tau_{i}: T \rightarrow T$ such that $t \mapsto t+i$ (if $t+i$ exists for every tone $t$ ). Obviously, in case of a homogeneous tone system, a transposition $\tau_{i}$ exists for every $i \in \delta[T \times T]$. In particular, an $n$-generated tone system allows a transposition by any interval of $I$, and we observe, that the set of all transpositions forms a transformation group canonically isomorphic to the interval group I.

A morphism from a tone system $\mathrm{T}=(T, \delta, I)$ to a tone system $\mathrm{T}^{\prime}=\left(T^{\prime}, \delta^{\prime}, I^{\prime}\right)$ is defined as a pair $\phi:=\left(\phi_{T}, \phi_{I}\right)$, consisting of a map $\phi_{T}: T \rightarrow T^{\prime}$ and a group homomorphism $\phi_{I}: \mathrm{I} \rightarrow \mathrm{I}^{\prime}$, such that for all $s, t \in T$ we have

$$
\phi_{I}(\delta(s, t))=\delta^{\prime}\left(\phi_{T}(s), \phi_{T}(t)\right) .
$$

If, in addition, $\phi_{T}$ and $\phi_{I}$ are bijections then $\phi$ is called an isomorphism (from $\mathrm{T}$ to $\left.\mathrm{T}^{\prime}\right)$. Every transposition $\tau$ induces via $\left(\tau, \mathrm{id}_{I}\right)$ an automorphism on T.

For every positive integer $n$, a freely $n$-generated tone system is always isomorphic to the tone system $\left(\mathbb{Z}^{n}, \delta, \mathbb{Z}^{n}\right)$ where $\delta(x, y):=y-x$ for all $x, y \in \mathbb{Z}^{n}$.

In the following we consider the 1-generated tone system $\mathrm{T}=(\mathbb{Z}, \delta, \mathbb{Z})$ and we fix a positive integer $\mathfrak{O} \in \mathbb{Z}_{+}$, which we consider as an interval called octave. Let $\mathbb{Z}_{\mathfrak{O}}$ denote the residue ring of integers modulo $\mathfrak{O}$ and let $\mathrm{T}_{\mathfrak{O}}:=\left(\mathbb{Z}_{\mathfrak{O}}, \delta_{\mathfrak{O}}, \mathbb{Z}_{\mathfrak{O}}\right)$ be the 1-generated algebraic tone system (where $\delta_{\mathfrak{O}}(x, y)$ denotes the difference $y-x$ in $\left.\mathbb{Z}_{\mathfrak{O}}\right)$. Following the language of musicology, $T_{\mathfrak{O}}$ is called a chroma system, and its elements are refereed to as chromas. More specifically, we will refer to $T_{\mathfrak{O}}$ as $\mathfrak{O}$-tone equal tempered chroma system, in short $\mathfrak{O}$-tet. The most commonly used of these are the 12-tet $\left(\mathrm{T}_{12}\right)$ and the 7-tet $\left(\mathrm{T}_{7}\right)$. 
Table 1. parameters describing concept lattices of harmonic forms.

\begin{tabular}{l||r|r|r} 
Group & \# of harmonic forms & \# of irreducibles (rows/columns) & \# of concepts \\
\hline $\mathbb{Z}_{1}$ & 2 & 1 & 2 \\
$\mathbb{Z}_{2}$ & 3 & 2 & 3 \\
$\mathbb{Z}_{3}$ & 4 & 3 & 4 \\
$\mathbb{Z}_{4}$ & 6 & 4 & 6 \\
$\mathbb{Z}_{5}$ & 8 & 6 & 9 \\
$\mathbb{Z}_{6}$ & 14 & 11 & 18 \\
$\mathbb{Z}_{7}$ & 20 & 13 & 42 \\
$\mathbb{Z}_{8}$ & 36 & 25 & 142 \\
$\mathbb{Z}_{9}$ & 108 & 39 & 1460 \\
$\mathbb{Z}_{10}$ & 188 & 73 & 9325 \\
$\mathbb{Z}_{11}$ & 352 & 112 & 1798542 \\
$\mathbb{Z}_{12}$ & & 212 & 208946771
\end{tabular}

The canonical group homomorphism $\phi_{\mathfrak{O}}: \mathbb{Z} \rightarrow \mathbb{Z}_{\mathfrak{O}}$ (which maps every integer $x$ to its residue modulo $\mathfrak{O}$, denoted by $\left.x_{\mathfrak{O}}\right)$ induces via $\left(\phi_{\mathfrak{O}}, \phi_{\mathfrak{O}}\right)$ a morphism from $T$ onto $T_{\mathfrak{O}}$. Every chord $X$ in $T$ is mapped to the chord $X_{\mathfrak{O}}:=\left\{x_{\mathfrak{O}} \mid x \in X\right\}$ in $T_{\mathfrak{D}}$, which will be called the harmony of $X$.

Chords and harmonies can efficiently be classified by the occurrence of intervals and chromas. In particular, two chords or harmonies have the same pattern if they are related by a transposition. The corresponding pattern classes we refer to as chordal forms or harmonic forms, respectively.

The degree of consonance or dissonance of a harmony is mostly influenced by its pattern.

Harmonic forms and their hierarchical order have been studied by RUdoLF WiLLE and other authors ([5]). Though the corresponding concept lattice for the 7-tet $\mathrm{T}_{7}$ has a nice diagram (see figure 1), the number of concepts of $\mathrm{T}_{\mathfrak{O}}$ is rapidly growing for increasing octave $\mathfrak{O}$. Table 1 shows some statistics about these concept lattices. For every chroma system there are printed the number of harmonic forms, the count of rows and columns in the formal context (describing the order of the harmonic forms), and the number of formal concepts in the corresponding concept lattice.

In other important chroma systems the hierarchical order of harmonic forms is too complex to be examined in the fashion above. Therefore, it is interesting to use other properties to clarify the structure of harmonic forms.

One important property is the interval structure of harmonic forms, since the intervals contained in a harmonic form have a major impact on their degree of consonance or dissonance. One example is shown in figure 2. Here, for the 7-tet a formal context is composed of the set of harmonic forms as objects, the set of intervals as attributes, and the interval occurrence as incidence relation. Also, in comparison with figure 1, this concept lattice is significantly simpler (but ordered oppositely).

The other corresponding lattices are relatively small too, as shown in table 2 , which enables us to have a view on the 12-tet lattice (fig. 3). 


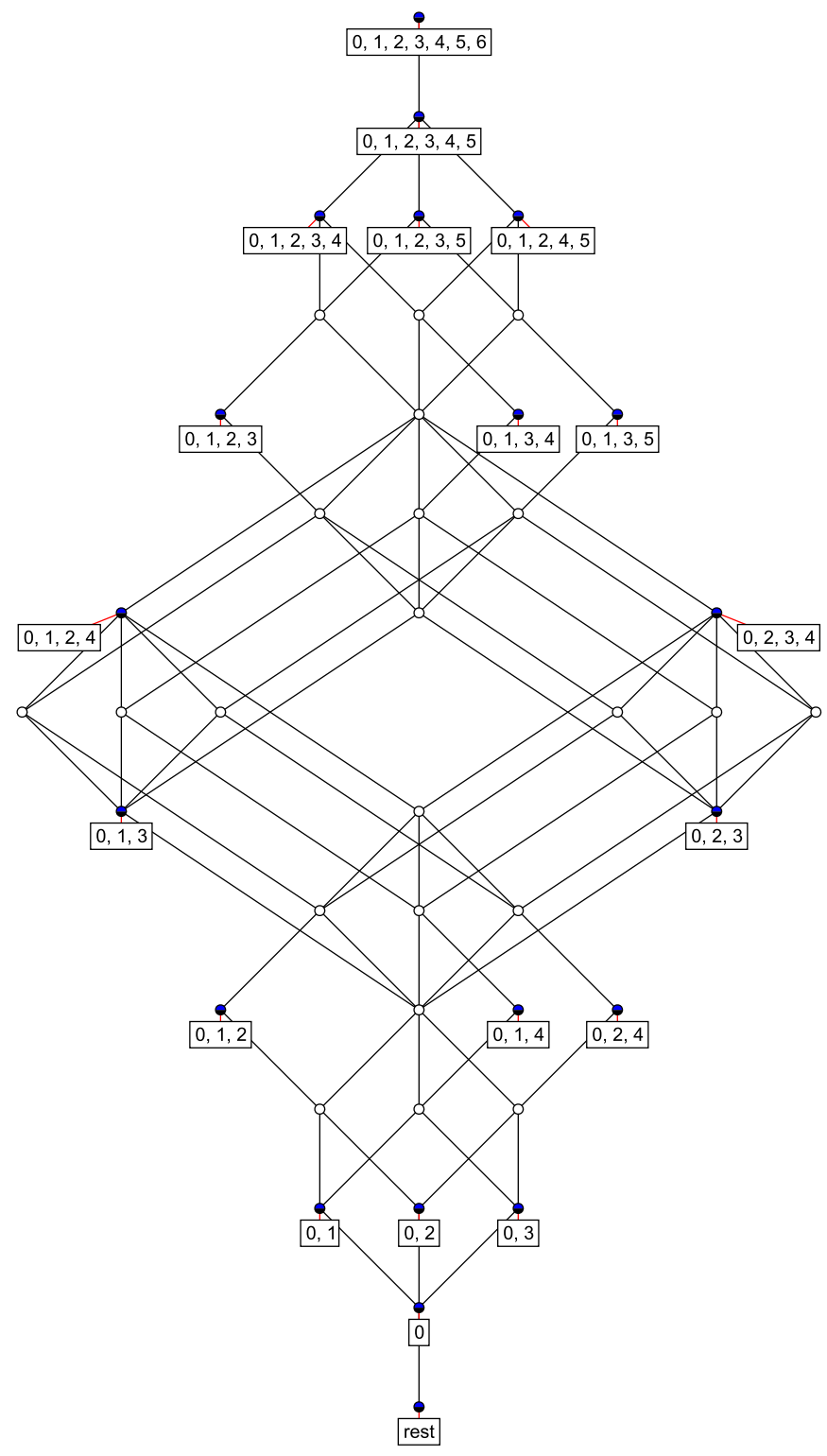

Fig. 1. Dedekind-McNeille completion of the order of harmonic forms in $\mathbb{Z}_{7}$. 


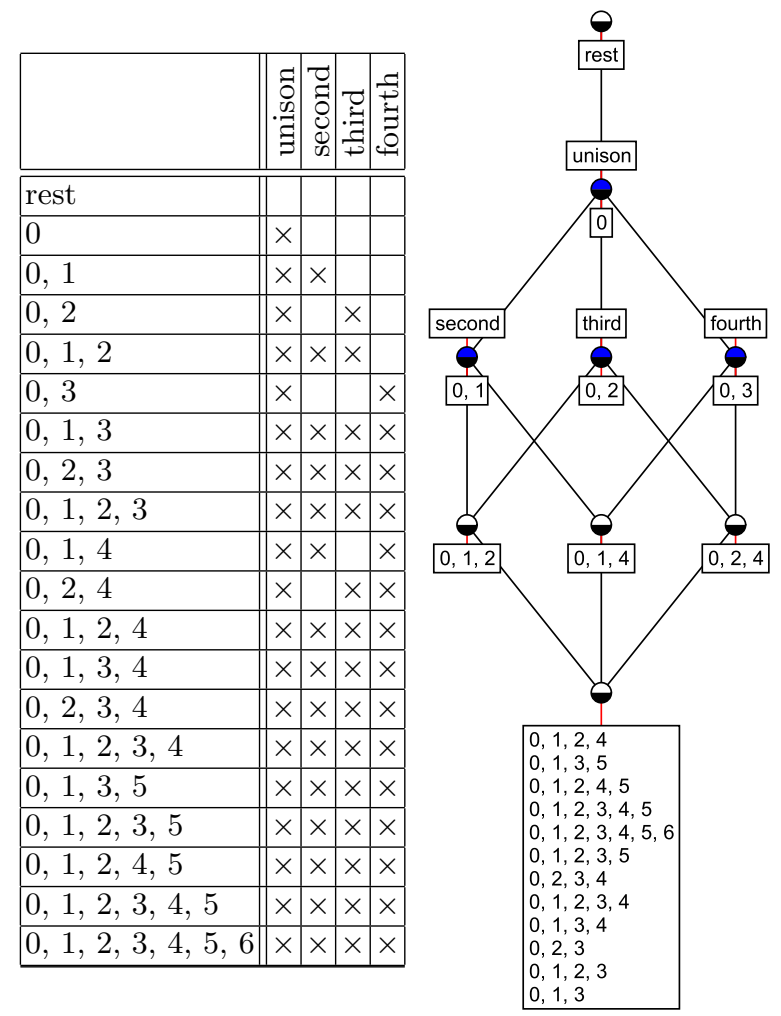

Fig. 2. Formal context and concept lattice which qualitatively describes the contained intervals of harmonic forms in the 7-tet

Table 2. Statistics of harmony interval concept lattices

Group \# of harmonic forms \# forms clar. \# forms red. \# intervals \# int. red. \#concepts

\begin{tabular}{l|rrrrrr}
\hline $\mathbb{Z}_{1}$ & 2 & 2 & 1 & 1 & 1 & 2 \\
$\mathbb{Z}_{2}$ & 3 & 3 & 2 & 2 & 2 & 3 \\
$\mathbb{Z}_{3}$ & 4 & 3 & 2 & 2 & 2 & 3 \\
$\mathbb{Z}_{4}$ & 6 & 5 & 3 & 3 & 3 & 5 \\
$\mathbb{Z}_{5}$ & 8 & 5 & 3 & 3 & 3 & 5 \\
$\mathbb{Z}_{6}$ & 14 & 7 & 5 & 5 & 4 & 7 \\
$\mathbb{Z}_{7}$ & 20 & 9 & 4 & 4 & 4 & 9 \\
$\mathbb{Z}_{8}$ & 36 & 12 & 6 & 5 & 5 & 13 \\
$\mathbb{Z}_{9}$ & 60 & 13 & 7 & 5 & 5 & 16 \\
$\mathbb{Z}_{10}$ & 108 & 20 & 6 & 6 & 6 & 33 \\
$\mathbb{Z}_{11}$ & 188 & 23 & 6 & 6 & 6 & 33 \\
$\mathbb{Z}_{12}$ & 352 & 32 & 7 & 6 & 7 & 65
\end{tabular}


Figures 2 and 3 are largely Boolean lattices (except the node named "rest"). It is also visible in figure 3 that not every node has a label. In the language of music this means that the intervals cannot be combined freely. They have to fulfil certain restrictions.

On the other hand, in this approach we consider neither the order nor the multiplicity of intervals. So with this method some sets of harmonic forms are identified. For example the major triad and the minor triad share the same label since they consist of a minor third, a major third and a fourth as chroma intervals.

In comparison with the concept lattices of the 7 -tet and the 12-tet, the ones of the 6 -tet and the 8-tet are less symmetric (figure 4). This means, the lattices have a more complex underlying structure.

\section{$3 \quad$ Analysis reflecting interval multiplicities}

The DedeKind-McNeILle completion of the ordering of harmonic forms does not reflect the notion of an interval. However, it describes a much finer granularity than given by the previously discussed type of lattice (derived from interval occurrence). The gap between these two lattice types can be filled by considering many-valued contexts, which reflect multiplicities of intervals within harmonic forms.

Figure 5 shows such a context in the 7 -tet. Its ordinary scaled version is shown in figure 6 and the corresponding concept lattice appears in figure 7. This lattice has more concepts than the one presented in figure 2 , but it contains less information than the one in figure 1 . Though the multiplicity of intervals is reflected by the lattice, their internal arrangement remains neglected. For example, the harmonic forms of $\{0,1,3\}$ and $\{0,2,3\}$ are different but share the same node. This impacts the musical interpretation, as some harmonic forms (like the major triad and the minor triad in the 12-tet) are indistinguishable.

Furthermore, the lattice of figure 7 has a very regular structure. In the upper part, the free distributive lattice with three generating elements is visible. The concepts below it form a typical configuration for all lattices of this family, which can be seen more clearly in the lattices investigated in the sequel.

After observing the beautiful structure provided by the 7-tet, we investigated higher orders in a similar fashion. Our suggestive approach was to draw the diagrams by ordering the intervals from left to right according to their sizes. For the 8-tet this can be seen in the left diagram of figure 8 .

Aiming for further insight, the following question arises: Which lattices allow nice diagrams and how can these diagrams be realised? To get a clue how to answer this question we focus again on the left diagram in figure 8. The right hand side of this diagram shows some interesting structural specialities: There are shorter chains from top to bottom of the lattice than on the left hand side. This phenomenon has the following cause: The concepts on the right hand side share the fifth (distance 4), which is an element of order 2 in the interval group. So these intervals occur only in pairs. This differs from the other intervals, which 


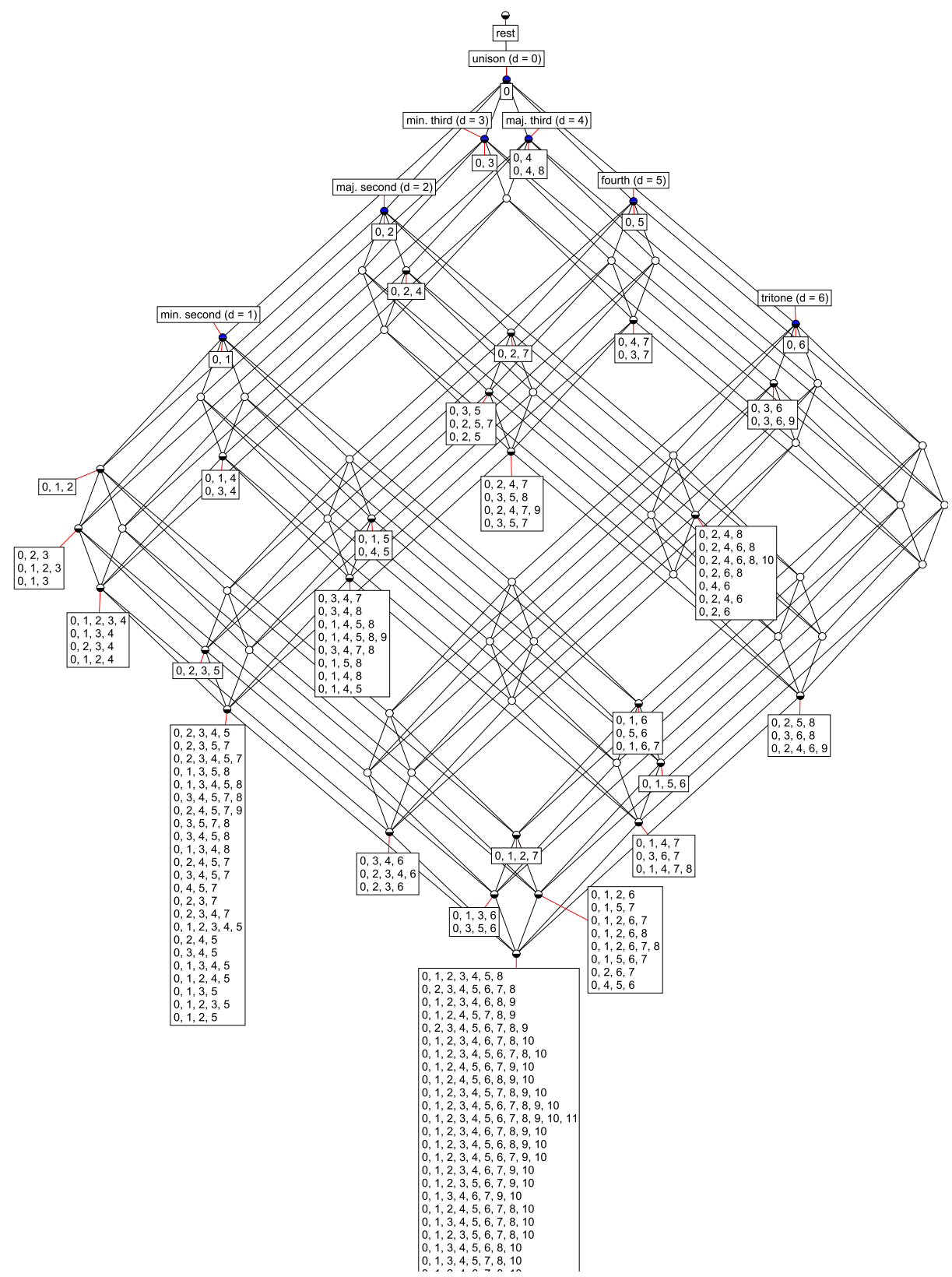

Fig. 3. Concept lattice qualitatively describing the contained intervals of harmonic forms in the 12-tet. The list of harmonic forms in the lower node is truncated. 

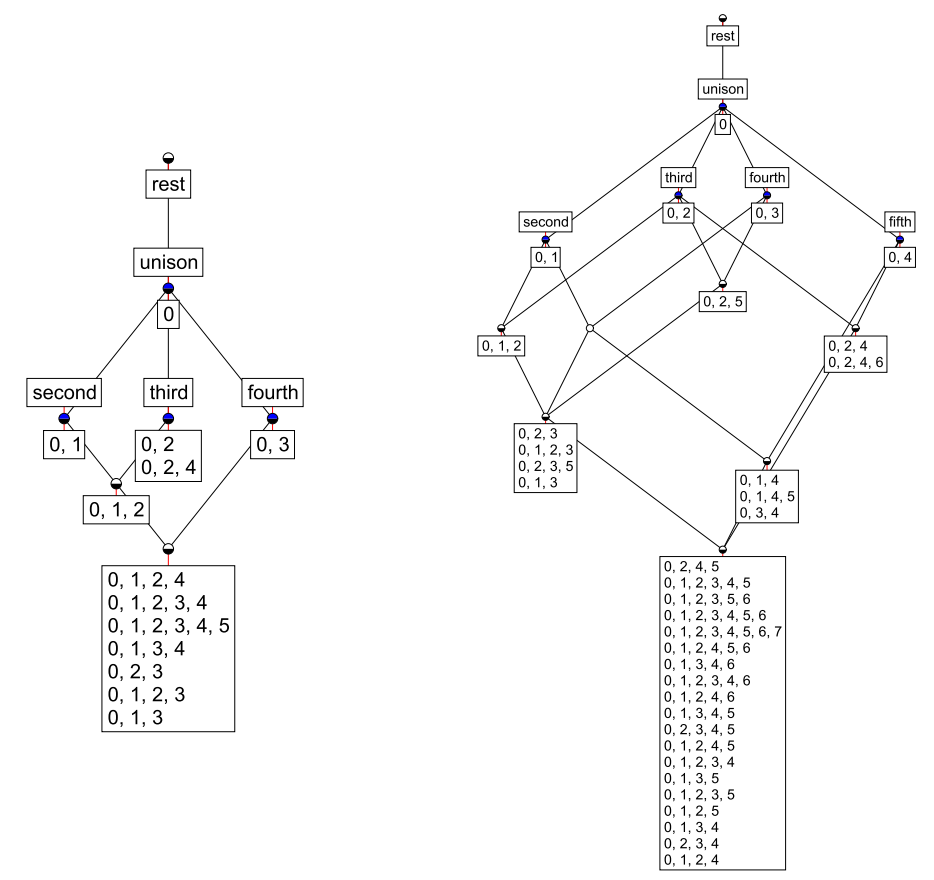

Fig. 4. Concept lattice qualitatively describing the contained intervals of harmonic forms in the 6-tet and the 8-tet

\begin{tabular}{|l||c|c|c|c|}
\hline pattern & unison & second & third & fourth \\
\hline \hline rest & & & & \\
\hline 0 & 1 & & & \\
\hline 0,1 & 2 & 1 & & \\
\hline 0,2 & 3 & 2 & 1 & \\
\hline $0,1,2$ & 2 & & & 1 \\
\hline 0,3 & 3 & 1 & 1 & 1 \\
\hline $0,1,3$ & 3 & 1 & 1 & 1 \\
\hline $0,2,3$ & 4 & 3 & 2 & 1 \\
\hline $0,1,2,3$ & 3 & 1 & & 2 \\
\hline $0,1,4$ & 3 & & 2 & 1 \\
\hline $0,2,4$ & 4 & 2 & 2 & 2 \\
\hline $0,1,2,4$ & 4 & 2 & 2 & 3 \\
\hline $0,1,3,4$ & 4 & 2 & 2 & 2 \\
\hline $0,2,3,4$ & 5 & 4 & 3 & 3 \\
\hline $0,1,2,3,4$ & 4 & 1 & 3 & 2 \\
\hline $0,1,3,5$ & 5 & 3 & 4 & 3 \\
\hline $0,1,2,3,5$ & 5 & 3 & 3 & 4 \\
\hline $0,1,2,4,5$ & 6 & 5 & 5 & 5 \\
\hline $0,1,2,3,4,5$ & 7 & 7 & 7 & 7 \\
\hline $0,1,2,3,4,5,6$ & & & & \\
\hline & & & 1 & \\
\hline
\end{tabular}

Fig. 5. Many-valued context describing the contained intervals of the 7-tet 


\begin{tabular}{|c|c|c|c|c|c|c|c|c|c|c|c|c|c|c|c|c|c|c|c|c|c|}
\hline & 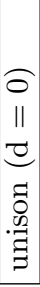 & 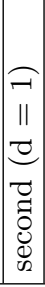 & 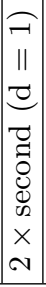 & 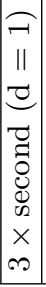 & 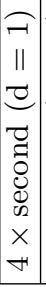 & $\begin{array}{c}-1 \\
\| \\
\| \\
0 \\
0 \\
0 \\
0 \\
0 \\
0 \\
0 \\
x \\
x \\
0\end{array}$ & 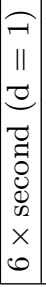 & 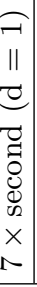 & 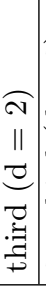 & 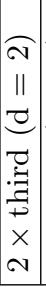 & 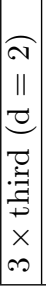 & 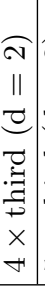 & 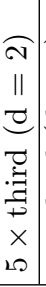 & 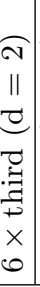 & 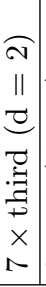 & 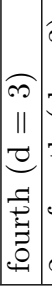 & 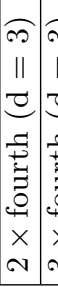 & 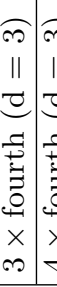 & 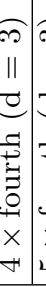 & 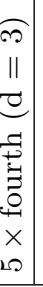 & \\
\hline rest & & & & & & & & & & & & & & & & & & & & & \\
\hline 0 & $x$ & & & & & & & & & & & & & & & & & & & & \\
\hline 0,1 & $x$ & $\times$ & & & & & & & & & & & & & & & & & & & \\
\hline 0,2 & $x$ & & & & & & & & $\times$ & & & & & & & & & & & & \\
\hline $0,1,2$ & $x$ & $x$ & $x$ & & & & & & $x$ & & & & & & & & & & & & \\
\hline 0,3 & $x$ & & & & & & & & & & & & & & & $x$ & & & & & \\
\hline $0,1,3$ & $\times$ & $\times$ & & & & & & & $\times$ & & & & & & & $x$ & & & & & \\
\hline $0,2,3$ & $x$ & $x$ & & & & & & & $x$ & & & & & & & $x$ & & & & & \\
\hline $0,1,2,3$ & $x$ & $x$ & $\times$ & $x$ & & & & & $\times$ & $x$ & & & & & & $x$ & & & & & \\
\hline $0,1,4$ & $x$ & $x$ & & & & & & & & & & & & & & $x$ & $x$ & & & & \\
\hline $0,2,4$ & $x$ & & & & & & & & $\times$ & $x$ & & & & & & $x$ & & & & & \\
\hline $0,1,2,4$ & $x$ & $x$ & $\times$ & & & & & & $x$ & $x$ & & & & & & $x$ & $\times$ & & & & \\
\hline $0,1,3,4$ & $x$ & $x$ & $\times$ & & & & & & $x$ & & & & & & & $x$ & $\times$ & $\times$ & & & \\
\hline $0,2,3,4$ & $x$ & $x$ & $x$ & & & & & & $\times$ & $x$ & & & & & & $x$ & $x$ & & & & \\
\hline $0,1,2,3,4$ & $x$ & $x$ & $\times$ & $\times$ & $x$ & & & & $\times$ & $x$ & $x$ & & & & & $x$ & $\times>$ & $\times$ & & & \\
\hline $0,1,3,5$ & $x$ & $x$ & & & & & & & $x$ & $x$ & $x$ & & & & & $x$ & $\times$ & & & & \\
\hline $0,1,2,3,5$ & $x$ & $x$ & $\times$ & $\times$ & & & & & $\times$ & $x$ & $\times$ & $x$ & & & & $x$ & $\times$ & $\times$ & & & \\
\hline $0,1,2,4,5$ & $x$ & $x$ & $\times$ & $\times$ & & & & & $\times$ & $\times$ & $\times$ & & & & & $\times$ & $\times$ & \begin{tabular}{l|l}
$\times$ \\
\end{tabular} & $\times$ & & \\
\hline $0,1,2,3,4,5$ & $x$ & $x$ & $x$ & $x$ & $x$ & $x$ & & & $\times$ & $x$ & $x$ & $\times$ & $x$ & & & $x$ & $x$ & \begin{tabular}{l|l}
$\times$ & $>$ \\
\end{tabular} & $x$ & $\times$ & \\
\hline $0,1,2,3,4,5,6$ & $x$ & $x$ & $\times$ & $\times$ & $\times$ & $\times$ & $x$ & $\times$ & $\times$ & $x$ & $\times$ & $\times$ & $\times$ & $\times$ & $\times$ & $\times$ & \begin{tabular}{l|l}
$\times$ & $>$ \\
\end{tabular} & \begin{tabular}{l|l}
$\times$ & $x$ \\
\end{tabular} & $\times$ & $\times$ & $\times x$ \\
\hline
\end{tabular}

Fig. 6. Scaled formal context quantitatively describing the contained intervals of harmony patterns in the 7 -tet 


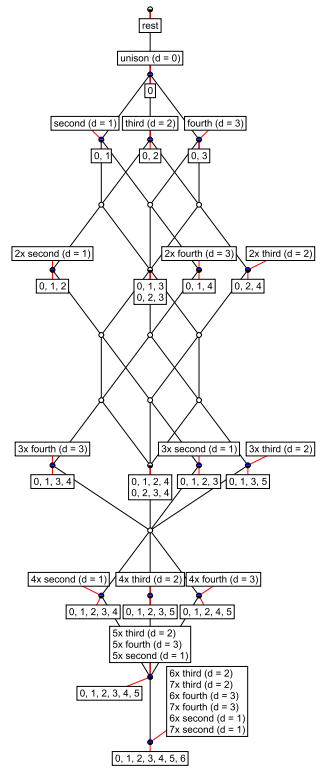

Fig. 7. Concept lattices of the context counting the intervals in the 7-tet
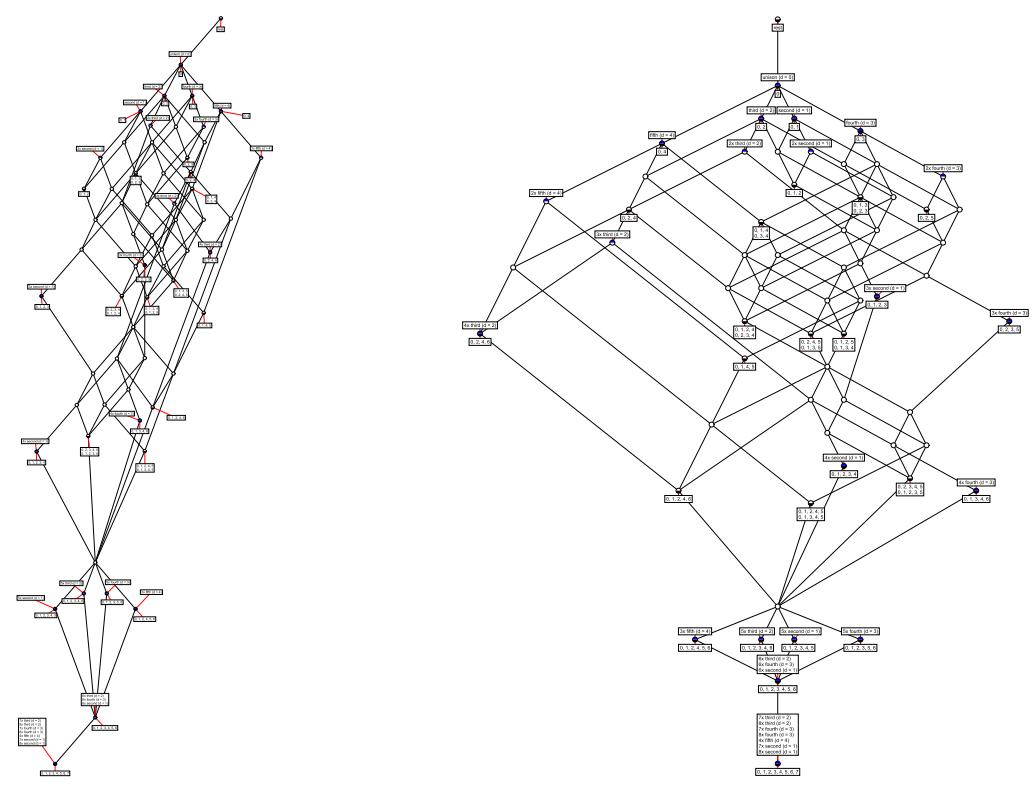

Fig. 8. Concept lattice describing the contained intervals in the 8-tet in two different chain decompositions 
can be combined more freely. For example the harmonic form represented by the harmony $\{0,1,2,3,4\}$ has only three (unordered) pairs of chromas containing a third, namely $\{0,2\},\{1,3\},\{2,4\}$.

Thus, divisibility of the group order has an impact on the (potential) layout and also the aesthetics of the generated diagram. On the other hand, harmonic forms of $\mathrm{T}_{4}$ can be embedded into $\mathrm{T}_{8}$ in various ways. The most important ones are defined by the mappings of chromas $f_{1}: t \mapsto t$ and $f_{2}: t \mapsto 2 t$. Each of these maps preserves to a reasonable extent the interval structure.

This suggests to rearrange the order of the intervals in the diagram to better unfold the lattice diagram. Coprime intervals (where coprime is meant in the number theoretic sense) should be positioned far apart from each other, while those with small greatest common divisor not equal to 1 , should be in close proximity. The unfolded lattice is shown on the right hand side of figure 8 .

It turns out that divisibility is the main property which makes the upper part of such a pattern lattice deviate from a product of chains. The lower part of the pattern lattice is of a significantly different shape. To discuss this, we point out the following general fact: In the chroma system $T_{\mathfrak{D}}$ intervals can be described as LEE distances (see [11]) between chromas.

The lowest point of such a diagram (as given in figure 8) represents the harmonic form of the complete chroma set $\mathbb{Z}_{\mathfrak{O}}$. Here, the LEE distances between unordered pairs of chromas form the set $\{0, \ldots,\lfloor\mathfrak{O} / 2\rfloor\}$. In the diagram the upper neighbour of the concept of the complete harmonic form represents an almost complete chroma set of size one less than $\mathbb{Z}_{\mathfrak{O}}$. That means, all intervals occur with the same frequency $\mathfrak{O}-2$. Next, selecting $\mathfrak{O}-2$ chromas, results in a harmonic form with two chromas omitted. Thus, each interval which ends in one of these two points will occur only $\mathfrak{O}-4$ times in the pattern. But there is one exception: The interval between the two deleted points has been counted twice. Consequently, this interval occurs (still) $\mathfrak{O}-3$ times in the harmonic form. Obviously, with an increasing number of deleted chromas, the number of intervals additionally vanishing, decreases further.

Because of the above, the lattice is divided into several levels of object concepts according to the number of chromas in the harmonic forms. This is not hard to understand since every harmonic form with $k$ chromas contains $\frac{k(k-1)}{2}$ intervals (where multiplicities are respected). The latter means that the concept of such a harmonic form has $\frac{k(k-1)}{2}+1$ attribute concepts of interval sets above it. Adding a chroma increases the number of interval concepts by $k$.

In case of example $T_{12}$, the above mentioned levels of harmonic forms become increasingly apparent towards the bottom part of the lattice $T_{12}$ (see figure 9). The right hand side of the diagram shows the "lightning rod chains", which result from the even group order leading to the pairwise occurrence of tritone intervals (distance 6) as described above.

The structure of the left hand side in the diagram of $\mathrm{T}_{12}$ is induced by the divisibility of $1,2,3$ and 4 . Though nesting can simplify the diagram in certain cases, the resulting lattices are still too complex for us to analyse. The reason is that every interval chain $<\frac{n}{2}$ generates all the levels described above. An 


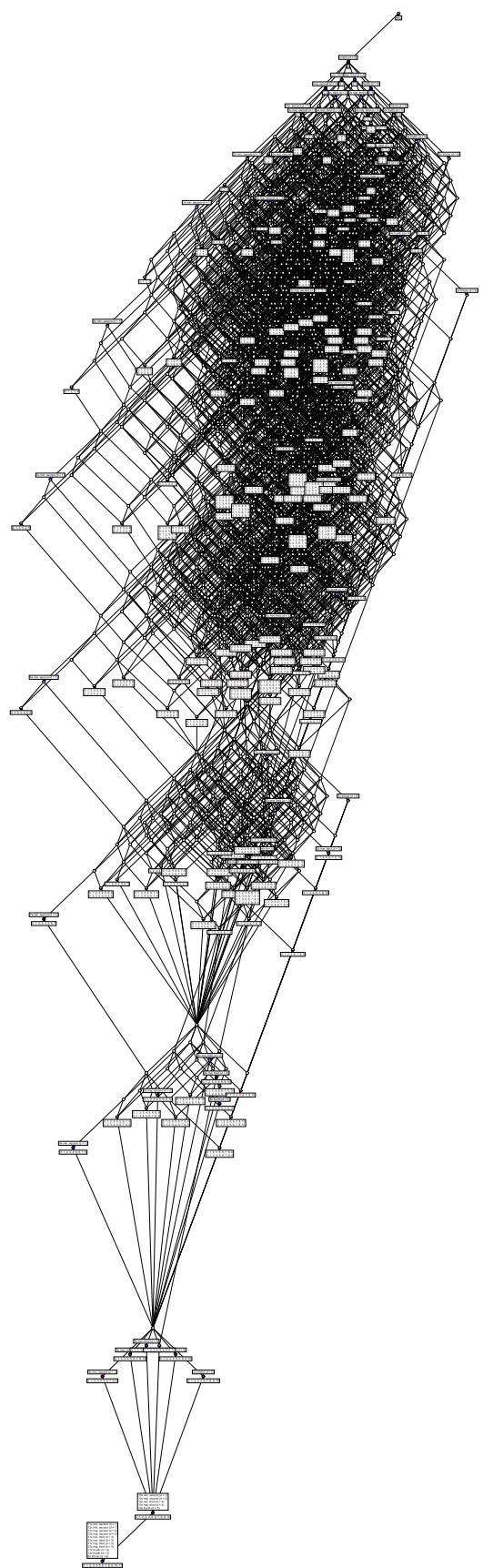

Fig. 9. Concept lattice of the harmony pattern vs. interval count context of the 12-tet 


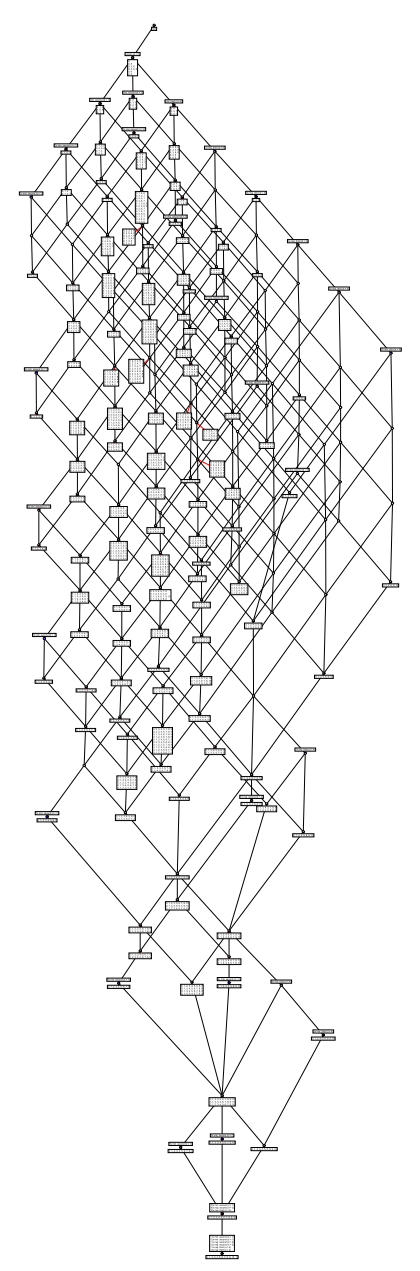

Fig. 10. Concept lattice created omitting all but the intervals 1,2 and 4 in the 12-tet

example of such a large projection which does not allow a sensible nesting is demonstrated in figure 10.

\section{Conclusions and further research topics}

The current work shows how one can overcome the obstacles of getting a meaningful interpretation of concept lattices of increasing complexity. In particular, we analyse concept lattices describing harmonic forms and their intervals in different ways, focusing on the 7-tet and the 12-tet.

For future analysis we propose that the information hidden in complex diagrams (e.g. as given in figure 9) may be used to further investigate the inherent structure of the concept lattice. 
Ongoing work is concerned with a description of the interval structure of a tone system and its influence on the structure of the concept lattices of harmonic forms in case of a totally ordered (or, more generally, lattice ordered) interval group.

Another extension of this work will be the investigation of tone systems with more complicated interval structures, for example the diatonic scale and LEONHARD EULER's Tonnetz.

This area of research also aims to have an impact on the further development of the tuning software Mutabor.

\section{References}

1. Mutabor team: Mutabor. Software http://www.math.tu-dresden.de/ mutabor/.

2. Ganter, B., Wille, R.: Formal concept analysis: mathematical foundations. Springer, Berlin (1999)

3. Wille, R., Wille-Henning, R.: Towards a semantology of music. In Priss, U., Polovina, S., Hill, R., eds.: ICCS. Volume 4604 of Lecture Notes in Computer Science., Springer (2007) 269-282

4. Wille, R., Wille-Henning, R.: Beurteilung von Musikstücken durch Adjektive: Eine begriffsanalytische Exploration. In: Von Intentionalität zur Bedeutung konventionalisierter Zeichen: Festschrift für Gisela Harras zum 65. Geburtstag. Volume Bd. 35. G. Narr, Tübingen (2006) 453

5. Wille, R.: Musiktheorie und Mathematik. In: Musik und Mathematik: Salzburger Musikgespräch 1984 unter Vorsitz von Herbert von Karajan. Springer-Verlag, Berlin (1985) 4-31

6. Wille, R.: Mathematische Sprache in der Musiktheorie. In: Jahrbuch Überblicke Mathematik 1980. Bibliographisches Institut, Mannheim (1980) 167-184

7. Mazzola, G., Göller, S., Müller, S.: The topos of music: geometric logic of concepts, theory, and performance. Birkhauser Verlag, Boston, MA (2002)

8. Wille, R.: Triadic concept graphs. In Mugnier, M.L., Chein, M., eds.: Conceptual Structures: Theory, Tools and Applications. Volume 1453 of Lecture Notes in Computer Science., Springer (1998) 194-208

9. Neumaier, W., Wille, R.: Extensionale Standardsprache in der Musiktheorie - eine Schnittstelle zwischen Musik und Informatik. In Hesse, H., ed.: Mikrotöne. Volume III. Edition Helbing edn. Helbing, Insbruck (1990) 139-167

10. Neumaier, W.: Was ist ein Tonsystem? : eine historisch-systematische Theorie der abendländischen Tonsysteme, gegründet auf die antiken Theoretiker Aristoxenos, Eukleides und Ptolemaios, dargestellt mit Mitteln der modernen Algebra. Volume 9. Lang, Frankfurt am Main (1986)

11. Lee, C.: Some properties of nonbinary error-correcting codes. Information Theory, IEEE Transactions on 4(2) (Jun 1958) 77-82 